\title{
Anisotropy studies with the Pierre Auger Observatory
}

\author{
Ugo Gregorio Giaccari ${ }^{1 *}$, for the Pierre Auger Collaboration ${ }^{2}$ \\ ${ }^{1}$ Instituto de Física, Universidade Federal do Rio de Janeiro, Ilha do Fundão, RJ, Brazil \\ ${ }^{2}$ Observatorio Pierre Auger, Av. San Martín Norte 304, 5613 Malargüe, Argentina \\ full author list: www.auger.org/archive/authors_2013_06.html
}

E-mail: ugoeif.ufrj.br

\begin{abstract}
We report recent results about the anisotropies of the arrival directions of cosmic rays detected by the surface array of the Pierre Auger Observatory. A first-harmonic analysis of the right ascension distribution is presented in a wide energy range above $10^{16} \mathrm{eV}$ and a possible consistency of the phase in ordered energy intervals is discussed. Constancy of the phase that may be observed even if the significances of the amplitudes are relatively small. The search of anisotropies is also discussed as a function of both declination and right ascension in several energy ranges above $10^{18} \mathrm{eV}$. A targeted search of neutron sources within the Galaxy is also presented.
\end{abstract}

The European Physical Society Conference on High Energy Physics -EPS-HEP2013

18-24 July 2013

Stockholm, Sweden

\footnotetext{
*Speaker.
} 


\section{Introduction}

The anisotropies at different energies in the arrival direction of the cosmic rays are key observables to address fundamental questions about the origin and the nature of these particles. It is not known if the break observed in the energy spectrum [1] around $\sim 4 \times 10^{18} \mathrm{eV}$ represents the transition from a galactic to an extra-galactic component in the cosmic ray flux [2] or the signature of a proton dominated extragalactic spectrum due to the production of $e^{ \pm}$pairs [3]. If the origin of the cosmic rays below the ankle energy is galactic, models predict large scale anisotropy patterns of few percent level or more depending on the galactic magnetic fields, cosmic ray composition and sources distribution. On the other hand if the transition occurs below the ankle and the cosmic rays above $1 \mathrm{EeV}\left(1 \mathrm{EeV}=10^{18} \mathrm{eV}\right)$ have a predominant extragalactic origin, the anisotropy in their angular distribution could be below $1 \%$ level. The behaviour of the large scale anisotropies at $\mathrm{EeV}$ energies is one important observable for discerning between these two scenarios.

Since the mean travel distance of relativistic neutrons is $9.2 \mathrm{kpc}(\mathrm{E} / \mathrm{EeV})$ and the radius of the Galaxy is approximately $15 \mathrm{kpc}$, galactic sources could be detected through neutrons with energy above $1 \mathrm{EeV}$. If such neutrons are present they would point back to their sources in the galactic disk, since unlike protons they are not bent by the magnetic fields.

The Pierre Auger Observatory [4] is located in Malargüe, Argentina $\left(35.2^{\circ} \mathrm{S}, 69.5^{\circ} \mathrm{W}\right)$ at an altitude of $1400 \mathrm{~m}$ asl. The Observatory uses two complementary and independent detection techniques for the measurement of air shower initiated by cosmic rays, a surface detector array (SD) and a fluorescence detector $(\mathrm{FD})$. The $\mathrm{SD}$ measures the lateral distribution of secondary particles at ground level and has a duty cycle of $100 \%$. It consists of an array of 1600 water Cherenkov detectors arranged in an hexagonal grid with a detector spacing of $1500 \mathrm{~m}$ covering a total area of $3000 \mathrm{~km}^{2}$. In order to enhance the capabilities of the Observatory by lowering its energy threshold 49 additional detectors [5] have been deployed with a spacing of $750 \mathrm{~m}$ covering an area of $25 \mathrm{~km}^{2}$. This allows to detect cosmic rays with energies down to $10^{16} \mathrm{eV}$.

The FD observes the atmosphere above the SD with 27 optical telescopes grouped in 5 buildings on the perimeter of the array. The FD measures the fluorescence light emitted by the excited atmospheric nitrogen after the passage of the charged particles of the shower. This allows for the observation of longitudinal development of the air shower during clear and moonless nights, resulting in a duty cycle of about $13 \%$. The FD provides an almost calorimetric measurement of the energy of the primary particles and high-quality events triggered independently by the SD and the FD are used for the energy calibration of the SD data [6].

\section{First Harmonic Analysis in Right Ascension}

The harmonic analysis of the right-ascension distribution (Rayleigh formalism [7]) is one of the most common techniques used to search for large scale anisotropies in the arrival directions of cosmic rays. This analysis benefits from the almost uniform exposure in right-ascension of the ground array, and does not require knowledge of the dependence of its exposure upon declination. The reconstructed amplitude of the first harmonic is proportional to the dipolar component in the equatorial plane and the corresponding phase gives us the direction of the maximum intensity.

The statistics accumulated by the Observatory allows to detect anisotropies at the $1 \%$ level, but 

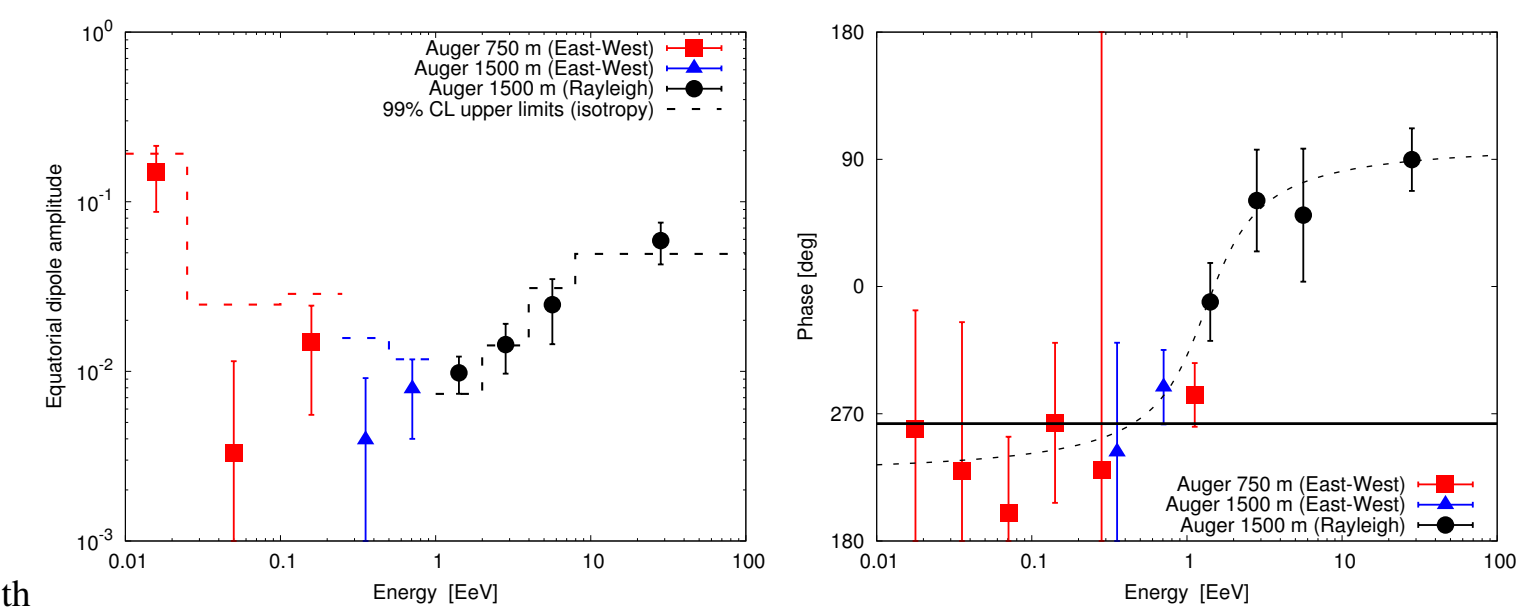

Figure 1: Left: Equatorial dipole amplitude as a function of energy. The results of the Rayleigh analysis are shown with black circles while the blue triangles and the red squares correspond to the analysis with EastWest method. Right: Phase of the first harmonic, the continuous line corresponds to the value $\phi=263^{\circ}$, that comes from a fit of the phase measured by the $750 \mathrm{~m}$ spacing array. The dotted line illustrates a smooth transition.

since the first harmonic modulations are quite small it is important to account for all the systematic effects of experimental and atmospheric origin that can produce spurious modulations in the counting rate. The directional exposure is controlled through the number of active stations, which is recorded every second and reflects the array growth as well as the dead periods of each detector. Atmospheric effects in the development of extensive air showers are accounted in the energy reconstruction to eliminate spurious modulations. Below $1 \mathrm{EeV}$ the systematic effects have a larger impact and the spurious variations in the counting rate are stronger. At these energies we use the differential East-West method [8], which does not require knowledge of the detection efficiency but has a reduced sensitivity to the first harmonic modulation.

Figure1 (left) shows the equatorial dipole amplitude in independent energy bins, the dashed line in the plot represents the upper values of the amplitude which may arise from fluctuations in an isotropic distribution at $99 \% \mathrm{CL}$. In the energy range 1-2 and 2-4 EeV the measured amplitudes have a probability to arise by chance from an isotropic distribution of about $0.03 \%$ and $0.9 \%$, while above $8 \mathrm{EeV}$ the probability is of $0.1 \%$. These measurements constitute interesting hints of anisotropies that will be important to investigate in the future with more data. The upper-limits on the equatorial dipole component derived from these measurements are relevant to constrain models in which the ankle is a signature of the galactic to extragalactic transition or in which a dominant galactic component extends to the highest energies [10]. The phase of the dipole as a function of the energy is shown in Figure1 (right). We note the intriguing possibility of a smooth transition from a common phase of $\alpha \simeq 270^{\circ}$ below $1 \mathrm{EeV}$ compatible with the right ascension of the Galactic Center $\alpha_{G C} \simeq 268.4^{\circ}$, and another phase $\alpha \simeq 100^{\circ}$ above $5 \mathrm{EeV}$. The phases are expected to be randomly distributed in the case of a pure isotropic distribution. This consistency in adjacent energy bins is expected with a smaller number of events than needed for the detection of amplitudes standing out significantly above the background noise in the case of a real underlying anisotropy. Given the posterior nature of this result no conclusion can be drawn, a prescription has 

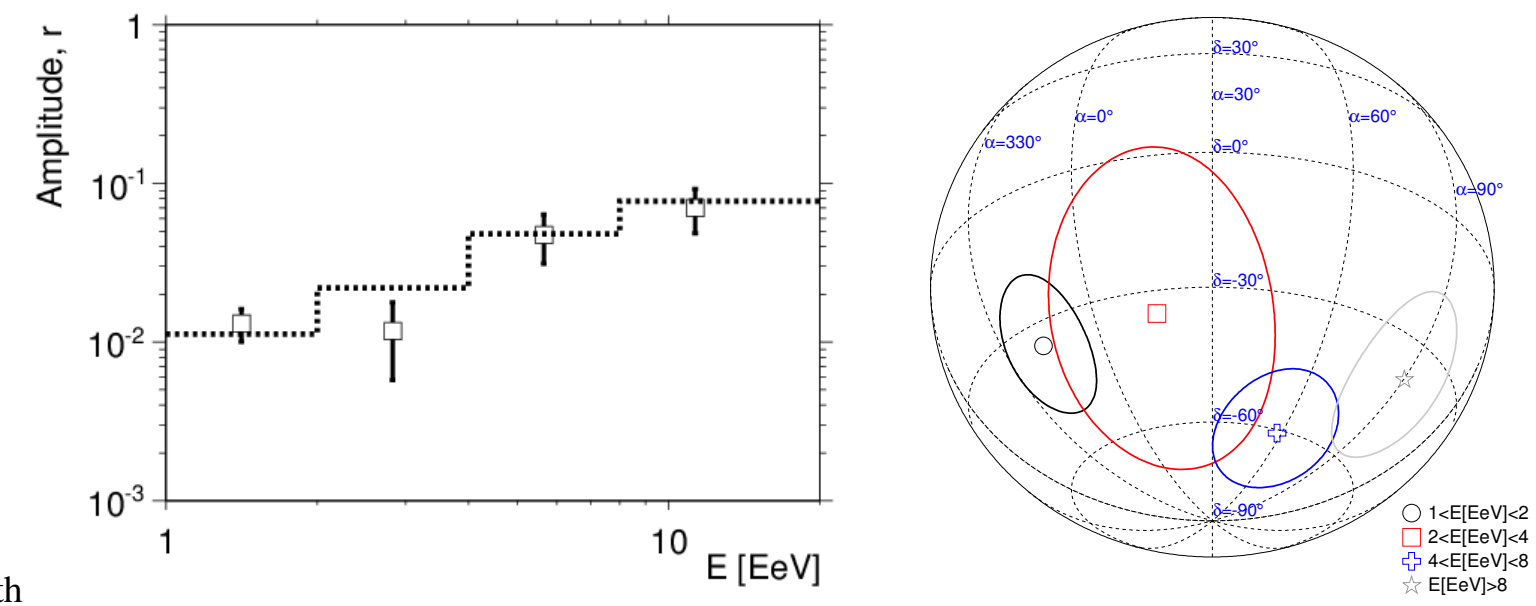

Figure 2: Left: Reconstructed amplitude of the dipole as a function of the energy. The dotted line stands for the $99 \%$ CL upper bounds on the amplitude that would result from fluctuations of an isotropic distribution. Right: Declination and right ascension of the reconstructed dipole with the corresponding uncertainties, as a function of the energy, in orthographic projection.

been designed and is currently operating to establish at 99\% CL the detection of a real transition.

\section{3D Multipolar Analysis}

Besides the harmonic analysis, which studies the arrival direction distribution only in right ascension, it is possible to study the anisotropies as a function of both right ascension and declination [11]. A thorough search for large scale anisotropies in terms of dipole and quadrupole moments above $10^{18} \mathrm{eV}$ has been published [12] with no significant deviations from isotropy reported. This analysis was recently updated [13], and we summarize here the results for a search of a dipole pattern. The search of anisotropies over the full celestial sphere requires accurate knowledge of the directional exposure both in right-ascension and declination. Systematic effects have been studied and different corrections have been applied to the data to take into account the influence of the geomagnetic field and atmospheric conditions on the energy estimation and the trigger efficiency. Figure2 (left) shows the reconstructed amplitude of the dipole above $10^{18} \mathrm{eV}$ in independent energy bins, the dotted line indicates the 99\% C.L. upper bounds on the amplitudes that would result from fluctuations of an isotropic distribution. Within the systematic uncertainties no significant deviation from isotropy is revealed. The results are compatible with the previous obtained with the harmonic analysis and show interesting hints of large scale anisotropies that will be important to scrutinise with independent data. Figure2 (right) shows the reconstructed directions of the dipole as a function of the energy in orthographic projection with the associated uncertainties. The right ascension of the dipole shows the same intriguing smooth alignment as in the previous Rayleigh analysis and it is interesting to note that all the reconstructed declinations are in the south hemisphere. As pointed out before both angles are expected to be randomly distributed in a case of a pure isotropic distribution. Assuming that the eventual anisotropic component of the angular distribution of the cosmic rays is dominated by dipole and quadrupole moments, upper limits on 
their amplitudes as a function of the energy have been obtained at the 99\% CL, see [13]. These bounds provide interesting constraints on the production of cosmic ray above $10^{18} \mathrm{eV}$, since they allow to challenge an origin from stationary sources densely distributed in the Galaxy and emitting predominantly light particles in all directions [14].

\section{Searches for Galactic Neutron Sources}

The mean decay length of neutrons in the EeV energy range is approximately $9.2 \mathrm{kpc}(\mathrm{E} / \mathrm{EeV})$, since the radius of the Galaxy is $\sim 15 \mathrm{kpc}$ galactic sources could be detected through neutrons with energy above $1 \mathrm{EeV}$. Unlike protons, neutrons have the advantage that are not deflected by the galactic magnetic fields. Neutrons produce air showers that are indistinguishable from those produced by protons, the signature of a neutron flux is a point like excess from a single celestial direction.

The Pierre Auger Collaboration has already reported a blind search of neutron sources over the full exposed sky that did not reveal any statistically significant excess [15] and allowed to place upper limits on the neutron flux at energies above $1 \mathrm{EeV}$. More recently a stacked search was performed based on catalogs of potential high-energy particle producers in the Galaxy [16]. Neutrons at EeV energies could be produced by interactions of ultra high-energy protons with the surrounding of the accelerator, gamma rays can also be generated in this scenario but they acquire a lesser fraction of the primary cosmic ray energy. For this reason candidate sources are expected to be strong gammaray emitters at $\mathrm{GeV}$ and $\mathrm{TeV}$ energies.

The search was performed in four energy ranges: $1 \leq \mathrm{E} / \mathrm{EeV}<2,2 \leq \mathrm{E} / \mathrm{EeV}<3, \mathrm{E} \geq 3 \mathrm{EeV}$, as well as $\mathrm{E} \geq 1 \mathrm{EeV}$. Different classes of potential cosmic ray sources have been used for this analysis, among them HESS sources, gamma- ray pulsars, low and high mass x-ray binaries, millisecond and standard radio pulsars, micro-quasars, and magnetars. Also the Galactic Plane and Galactic Center were considered. To search for the presence of an excess, a $p$-value has been assigned for each target in a set, defined as the Poisson probability to obtain a number of events greater than or equal to the one that was actually observed. The size of each target region was chosen in terms of the average angular resolution corresponding to its declination in the energy range considered. The stacked measure for a set of targets was then determined as the fraction of simulations in which the product of $p$-values is less than or equal to the same product obtained using the actual data. These stacked results reveal no significant excess from any of the target sets. Neutron flux and energy flux upper-limits at 95\% CL have been derived for each target. The energy flux upper limit has been computed assuming an $E^{-2}$ neutron spectra above $1 \mathrm{EeV}$. In Table 1 we show the neutron flux and energy flux upper limits for the targets with the smallest $p$-value in each of the potential classes of neutron sources, the observed and expected number of events and the coordinates of the targets. The values reported in this table are only for the energy range $\mathrm{E} \geq 1 \mathrm{EeV}$ which corresponds to the inclusive range containing all the other ones. Also listed is $p^{*}=1-(1-p)^{N}$, that gives the $p$-value of the target penalized for multiple trials, where $p$ is the original $p$-value and $N$ the number of targets in the set. The $p$-values for all the stacks and energy ranges considered and for the individual targets, once penalized for the multiple trials, are all larger than $2 \%$ which constitute no evidence for neutron fluxes originating from them. 


\begin{tabular}{|c|c|c|c|c|c|c|c|c|}
\hline Stack & $\mathrm{RA}\left[{ }^{\circ}\right]$ & $\operatorname{DEC}\left[^{\circ}\right]$ & Obs & Exp & $\begin{array}{l}\text { Flux U.L. } \\
{\left[\mathrm{km}^{-2} \mathrm{yr}^{-1}\right]}\end{array}$ & $\begin{array}{l}\text { E-Flux U.L. } \\
{\left[\mathrm{eV} \mathrm{cm}^{-2} \mathrm{~s}^{-1}\right]}\end{array}$ & $p$-value & $p^{*}$ \\
\hline Reg. PSRs & 267.44 & -56.09 & 249 & 204 & 0.0161 & 0.117 & 0.0012 & 0.78 \\
\hline msec PSRs & 270.46 & -14.29 & 174 & 146 & 0.0156 & 0.114 & 0.014 & 0.70 \\
\hline$\gamma$-ray PSRs & 195.60 & -32.95 & 222 & 191 & 0.0146 & 0.107 & 0.017 & 0.72 \\
\hline LMXB & 129.35 & -42.90 & 238 & 208 & 0.0135 & 0.0983 & 0.023 & 0.96 \\
\hline HMXB & 249.77 & -46.70 & 237 & 208 & 0.0129 & 0.0945 & 0.028 & 0.88 \\
\hline HESS & 284.58 & 2.09 & 101 & 80.6 & 0.0155 & 0.113 & 0.016 & 0.61 \\
\hline Microquasars & 288.75 & 10.08 & 68 & 53.4 & 0.0161 & 0.118 & 0.030 & 0.33 \\
\hline Magnetars & 248.97 & -47.59 & 224 & 209 & 0.00992 & 0.0724 & 0.15 & 0.88 \\
\hline G. Center & 266.40 & -28.94 & 178 & 186 & 0.0062 & 0.045 & 0.73 & - \\
\hline G. Plane & \multicolumn{2}{|c|}{ Galactic lat. $=0^{\circ}$} & 15488 & 15600 & - & - & 0.81 & - \\
\hline
\end{tabular}

Table 1: List of targets with smallest $p$-value in each target set for the energy range $\mathrm{E} \geq 1 \mathrm{EeV}$. The upper limits are derived at 95\% C.L.

\section{Conclusion}

We summarized recent anisotropy searches with data from the Pierre Auger Observatory. Interesting hints of large-scale anisotropy have been observed above $10^{18} \mathrm{eV}$ and will be scrutinized with more data. An intriguing smooth transition of the phase of the first-harmonic modulation in right-ascension has been observed, from a constant value compatible with the direction toward the Galactic Center below $1 \mathrm{EeV}$ to a different constant value around $100^{\circ}$ above $5 \mathrm{EeV}$. It is worth noting that the phase analysis can be more sensitive to reveal an anisotropy than the detection of an amplitude. A test with independent data is currently running to investigate the behaviour of the phase. We also summarized a search for neutron sources, based on catalogs of potential highenergy particle producers in the Galaxy. Upper bounds on neutron fluxes were derived, that place constraints on models of galactic sources of cosmic rays. 


\section{References}

[1] The Pierre Auger Collaboration, Phys. Lett. B (685) (2010) 239

[2] A. M. Hillas, Ann. Rev. Astr. Astrophys. 22 (1984) 425

[3] V. S. Berezinsky, A. Z. Gazizov and S. I. Grigorieva, Phys. Rev. D 74 (2006) 617625

[4] The Pierre Auger Collaboration, Nucl. Instr. and Meth. A (523) (2004) 50

[5] I. Maris for The Pierre Auger Collaboration Proc. 32 ICRC, Beijing, China (2011) arXiv:1107.4807.

[6] V. Verzi for The Pierre Auger Collaboration Proc. 33 ICRC, Rio de Janeiro, Brazil (2013) arXiv:1307.5059

[7] J. Linsley, Phys. Rev. Lett. 34 (1975) 1530

[8] R. Bonino et al., Astrophys. J. 67 (2011) 738

[9] The Pierre Auger Collaboration, Astropart. Phys. 34 (2011) 627

[10] I. Sidenik for The Pierre Auger Collaboration Proc. 33 ICRC, Rio de Janeiro, Brazil (2013) arXiv:1307.5059

[11] P. Billoir and O. Deligny, JCAP 02 (2008) 009

[12] The Pierre Auger Collaboration, ApJS 203 (2012) 34

[13] R. M. De Almeida for The Pierre Auger Collaboration Proc. 33 ICRC, Rio de Janeiro, Brazil (2013) arXiv:1307.5059

[14] The Pierre Auger Collaboration, ApJL 762 (2012) L13

[15] The Pierre Auger Collaboration, ApJ 760 (2012) 148

[16] F. Salesa Greus for The Pierre Auger Collaboration Proc. 33 ICRC, Rio de Janeiro, Brazil (2013) arXiv:1307.5059 